\title{
Electrically tunable all-dielectric optical metasurfaces based on liquid crystals
}

Andrei Komar, Zheng Fang, Justus Bohn, Jürgen Sautter, Manuel Decker, Andrey Miroshnichenko, Thomas Pertsch, Igal Brener, Yuri S. Kivshar, Isabelle Staude, and Dragomir N. Neshev

Citation: Appl. Phys. Lett. 110, 071109 (2017); doi: 10.1063/1.4976504

View online: http://dx.doi.org/10.1063/1.4976504

View Table of Contents: http://aip.scitation.org/toc/apl/110/7

Published by the American Institute of Physics

\section{Articles you may be interested in}

Ultra-compact visible chiral spectrometer with meta-lenses

APL Photonics 2, 036103 (2017); 10.1063/1.4974259

Reconfigurable conversions of reflection, transmission, and polarization states using active metasurface Applied Physics Letters 110, 121901 (2017); 10.1063/1.4979033

Electrically driven and electrically tunable quantum light sources

Applied Physics Letters 110, 071102 (2017); 10.1063/1.4976197

Electrically tunable diffraction efficiency from gratings in Al-doped $\mathrm{ZnO}$

Applied Physics Letters 110, 071110 (2017); 10.1063/1.4976573

Tamper indicating gold nanocup plasmonic films

Applied Physics Letters 110, 071101 (2017); 10.1063/1.4975936

All-dielectric phase-change reconfigurable metasurface

Applied Physics Letters 109, 051103 (2016); 10.1063/1.4959272

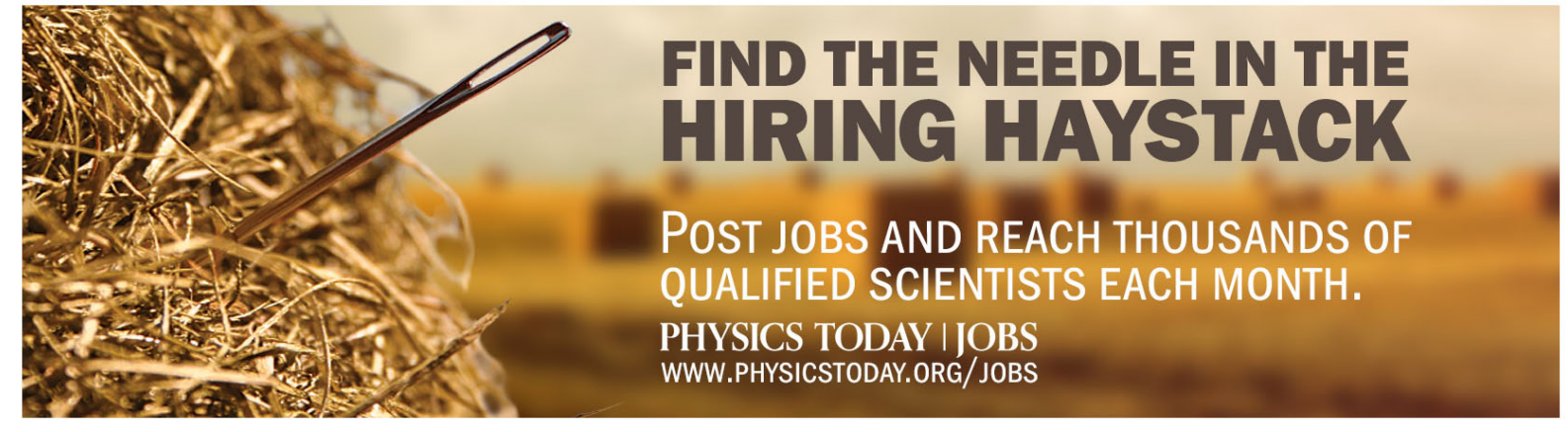




\title{
Electrically tunable all-dielectric optical metasurfaces based on liquid crystals
}

\author{
Andrei Komar, ${ }^{1}$ Zheng Fang, ${ }^{1}$ Justus Bohn, ${ }^{2}$ Jürgen Sautter, ${ }^{1}$ Manuel Decker, ${ }^{1}$ \\ Andrey Miroshnichenko, ${ }^{1}$ Thomas Pertsch, ${ }^{2}$ Igal Brener, ${ }^{3}$ Yuri S. Kivshar, ${ }^{1}$ \\ Isabelle Staude, ${ }^{1,2}$ and Dragomir N. Neshev ${ }^{1, a)}$ \\ ${ }^{1}$ Nonlinear Physics Centre and Centre for Ultrahigh bandwidth Devices for Optical Systems (CUDOS), \\ Research School of Physics and Engineering, The Australian National University, Canberra, 2601 ACT, \\ Australia \\ ${ }^{2}$ Institute of Applied Physics, Abbe Center of Photonics, Friedrich Schiller University Jena, 07745 Jena, \\ Germany \\ ${ }^{3}$ Center for Integrated Nanotechnologies, Sandia National Laboratories, Albuquerque, New Mexico 87185, \\ USA
}

(Received 12 October 2016; accepted 25 January 2017; published online 15 February 2017)

\begin{abstract}
We demonstrate electrical tuning of the spectral response of a Mie-resonant dielectric metasurface consisting of silicon nanodisks embedded into liquid crystals. We use the reorientation of nematic liquid crystals in a moderate applied electric field to alter the anisotropic permittivity tensor around the metasurface. By switching a control voltage "on" and "off," we induce a large spectral shift of the metasurface resonances, resulting in an absolute transmission modulation of up to $75 \%$. Our experimental demonstration of voltage control of dielectric metasurfaces paves the way for new types of electrically tunable metadevices, including dynamic displays and holograms. Published by AIP Publishing. [http://dx.doi.org/10.1063/1.4976504]
\end{abstract}

All-dielectric metasurfaces have proven to be a versatile tool for lossless manipulation of optical wavefronts, ${ }^{1-8}$ spectrum, ${ }^{9-13}$ and polarization. ${ }^{6,14}$ These high transmission efficiencies, derived from the lossless nature of the optical resonances in high refractive index dielectric nanoparticles, have spurred research into a wide range of applications, ${ }^{15,16}$ including lenses, beam deflectors, and holograms. However, these application prospects would broaden a lot further if the optical properties of the metasurface could be dynamically altered by an external control parameter, enabling applications such as flat lenses with dynamic focus, active beam steering, and holographic optical elements. Dielectric metasurfaces deriving their functionality from strong localized electric and magnetic Mie-type ${ }^{17-19}$ resonances of designed high-refractive index nanoparticles are particularly suited for achieving dynamic behaviour as their resonant optical properties are associated with a strong spectral dispersion, which can be tailored at will by the metasurface design. Several realizations of active tuning of the resonance positions of Mie-resonant dielectric metasurfaces were presented recently. ${ }^{20-23}$ The first demonstration used the temperature dependent change of the refractive index tensor of a nematic liquid crystal (LC) covering the silicon nanoresonators. ${ }^{20}$ Another work proposed to use the electric-field induced reorientation of LCs for tuning of all-dielectric metasurfaces; ${ }^{24}$ however, this effect was only theoretically studied. More recently, resonance tuning was demonstrated based on mechanical stretching of a flexible matrix into which the dielectric nanoresonators are embedded. ${ }^{21}$ This method was also employed to realize a dielectric metasurface lens with adjustable focal length. ${ }^{22}$ Tuning of a dielectric metasurface

\footnotetext{
${ }^{\text {a) }}$ Author to whom correspondence should be addressed. Electronic mail: Dragomir.Neshev@anu.edu.au
}

using the amorphous-crystalline transition in a chalcogenide phase-change material was also achieved. ${ }^{23}$ While all these methods can allow for large tuning ranges, they employ slow control mechanisms, namely, temperature change, ${ }^{20}$ mechanical stretch, ${ }^{21}$ and raster-scanning of a laser beam; ${ }^{23}$ then they are rather cumbersome and unlikely to find their way into practical applications. Ultrafast all-optical switching of Mie-resonant silicon nanostructures was also demonstrated; ${ }^{25-27}$ however, these effects are weak even at high optical intensities.

Here, we demonstrate an active control of the spectral response of Mie-resonant silicon metasurfaces using voltageinduced reorientation of the anisotropic nematic LC in a moderate electric field. We achieve a large tuning range of $50 \mathrm{~nm}$ and $75 \%$ absolute transmission change at $\lambda \approx 1550 \mathrm{~nm}$ by application of an $\mathrm{AC}$ bias voltage of $70 \mathrm{~V}$ at $1 \mathrm{kHz}$. In addition, while a dynamic phase shift of a reflecting metasurface consisting of a metal and a conducting oxide was previously measured by Huang et al., ${ }^{28}$ here we directly retrieve the tuning of the transmittance phase across the Mie-type resonance of a dielectric metasurface.

In our work, we use an all-dielectric metasurface composed of silicon nanodisks integrated into an LC cell, as shown in Figs. 1(a) and 1(b). Being composed of crystalline silicon, the nanodisks exhibit very low absorption losses at wavelengths above the silicon electronic bandgap around $1.1 \mu \mathrm{m}$ and support strong electric and magnetic dipolar Mietype resonances in the telecommunication spectral range, which can be tailored by adjusting the disk height, diameter, or lattice constant of the metasurface. ${ }^{4,9}$ Furthermore, such metasurfaces can be designed to operate as highly transparent Huygens' metasurfaces by bringing their electric and magnetic dipole resonances into spectral overlap. ${ }^{3,4}$ In this work, however, we choose the parameters such that the electric and 
(a)

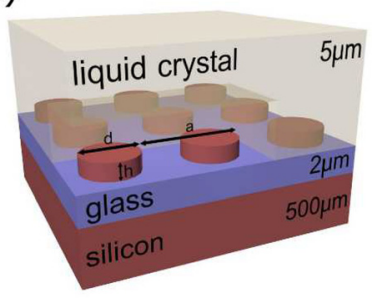

(b)

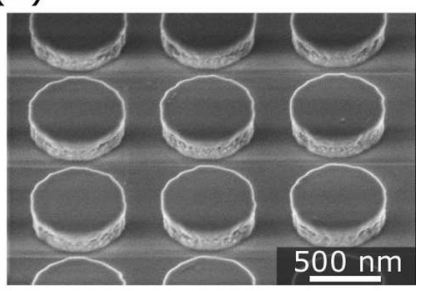

(c)

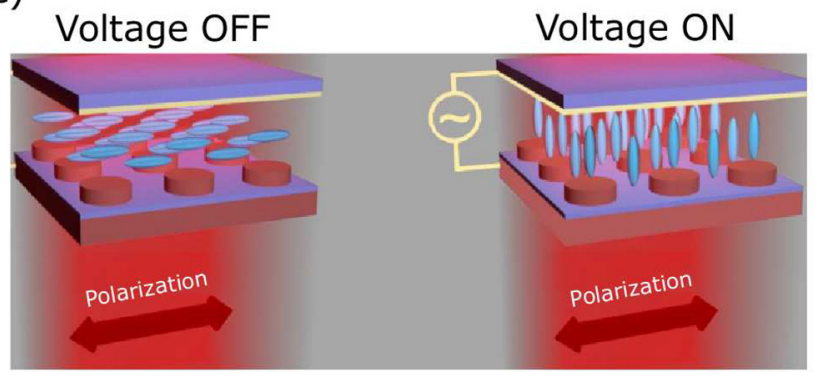

FIG. 1. (a) Sketch of a silicon nanodisk metasurface integrated into the LC cell. (b) SEM image of a fabricated silicon nanodisk metasurface. (c) Schematic of the LC alignment for no applied voltage ("off" case) and for the case when a moderate voltage is applied between the two electrodes of the LC cell ("on" case). The red arrow indicates the polarization of the incident light.

the magnetic resonances are well separated in order to clearly reveal and evaluate the resonance shifts induced by the voltage controlled LC realignment.

The fabrication of the metasurfaces is performed using electron-beam lithography on silicon-on-insulator wafers, followed by reactive ion etching and residual resist removal with an oxygen plasma, as described in detail in our previous work. ${ }^{8,9}$ Fig. 1(b) shows a scanning electron microscopy (SEM) image of a typical nanodisk metasurface. In our experiments, the nanodisks have a height of $h=220 \mathrm{~nm}$ and a diameter of $d=606 \mathrm{~nm}$. They are arranged in a squarearray with a lattice constant of $a=909 \mathrm{~nm}$. The dimensions of the disks are chosen such that the electric and magnetic dipolar resonances occur in the telecommunication wavelength range. The fabricated metasurface is then integrated into an LC cell in such a way that the silicon nanodisks are fully embedded into the LC. We employ the nematic LC Merck Licristal E7, which is widely used in display technologies and exhibits high birefringence $(\sim 0.2)$, with $n_{e}=1.7$ and $n_{0}=1.51$ at room temperature. ${ }^{29}$ The LC cell is constructed by sandwiching the LC between the metasurface wafer and a glass substrate coated with indium-tin-oxide (ITO) and with a brushed layer of Nylon-6 in 2,2,2-trichloroethanol. A suitable spacer material is employed to fix the (inside) thickness of the LC cell to $5 \mu \mathrm{m}$. The brushed Nylon-6 layer induces a preferred alignment direction of the LC. The ITO layer renders the substrate conductive for use as an electrode. In this way, a bias voltage can be applied between the ITO electrode and the silicon handle wafer of the metasurface sample. Although we do not additionally control the alignment of the LC on the metasurface, without an applied voltage, the LC alignment induced by the brushed Nylon-6 layer is approximately sustained throughout the LC cell (see Fig. 1(c), voltage “off”). This was verified by a strong optical anisotropy of the metasurface embedded into the LC without applied voltage. The application of a bias voltage between the two electrodes reorientates the LC molecules perpendicular to the metasurface (see Fig. 1(c), voltage "on"). As common for LC cells, we choose an AC bias voltage $(1 \mathrm{kHz})$, which is common in the modern LC industry standards and allows to avoid current flows, liquid crystal orientation instabilities, heating, and other detrimental effects associated with dc fields. ${ }^{30,31}$ The small thickness of the LC cell allows for strong reorientation of the LC in the vicinity of silicon disks.

In order to investigate the effect of LC reorientation on the optical properties of the metasurface, we numerically calculate the transmittance spectra of the silicon nanodisk metasurface covered by an ideal uniformly distributed nematic LC. We use the software package COMSOL Multiphysics and represent the $\mathrm{LC}$ as a homogeneous anisotropic dielectric medium with $n_{e}=1.7$ and $n_{0}=1.51 .^{29}$ The refractive index of silicon disks is 3.5 and silica substrate is 1.45 . The angle between the metasurface plane and the LC anisotropy axis is denoted with $\Theta_{L C}$, with $\Theta_{L C}=0^{\circ}$ being equivalent to the voltage "off" case and $\Theta_{L C}=90^{\circ}$ to the voltage "on" case (see also Fig. 2(a)). The polarization of the incoming field is parallel to the anisotropy axis of the LC in the "off" case. Thereby, the extraordinary wave is excited during the "off" state and the ordinary wave during the "on" state. To model the reorientation of the LC in the externally applied electric field, we tune the angle $\Theta_{L C}$ from $0^{\circ}$ to $90^{\circ}$ in steps of $10^{\circ}$. These results are shown in Fig. 2(a). The white lines highlight the spectral positions of the two resonances of interest. We observe a red-shift of the magnetic dipole resonance and a blue-shift of the electric dipole resonance with increasing angle $\Theta_{L C}$. Thus, the two resonances, having a clear spectral separation in the "off" case, move closer together for the LC realignment. Figure 2(b) shows the transmittance spectra for the ideal "on" and "off" cases for comparison with experimental data.

Next, using a home-built white-light spectroscopy setup, we measure the linear-optical transmittance spectra of the metasurface integrated into the LC cell. We use an unstructured etched area of the sample inside the same LC cell as a reference. A separate reference spectrum was taken for each applied voltage. Other Fabry-Perot artifacts induced by the layered structure of the system are avoided using the procedure described by Chong et al. $^{4}$ generalised to the layer structure of the LC cell. The results for the "off" case and for an applied AC bias voltage of $70 \mathrm{~V}$ at $1 \mathrm{kHz}$ are shown in Fig. 3(a). In the "off" case, we can clearly identify two pronounced transmission minima corresponding to the electric and magnetic dipole resonances of the silicon metasurface. Upon application of the bias voltage, the predicted red-shift of the magnetic dipole resonance and the blue-shift of the electric dipole resonance of the silicon metasurface are clearly observed and in good qualitative agreement with numerical expectations. Quantitatively, the observed shifts of $29 \mathrm{~nm}$ for the electric and $55 \mathrm{~nm}$ for the magnetic resonance are smaller in the experiment compared with numerical spectra $(69 \mathrm{~nm}$ for the electric and $61 \mathrm{~nm}$ for the magnetic resonance). Furthermore, we find deviations in the exact positions of the resonances both for the "off" case and 
(a)
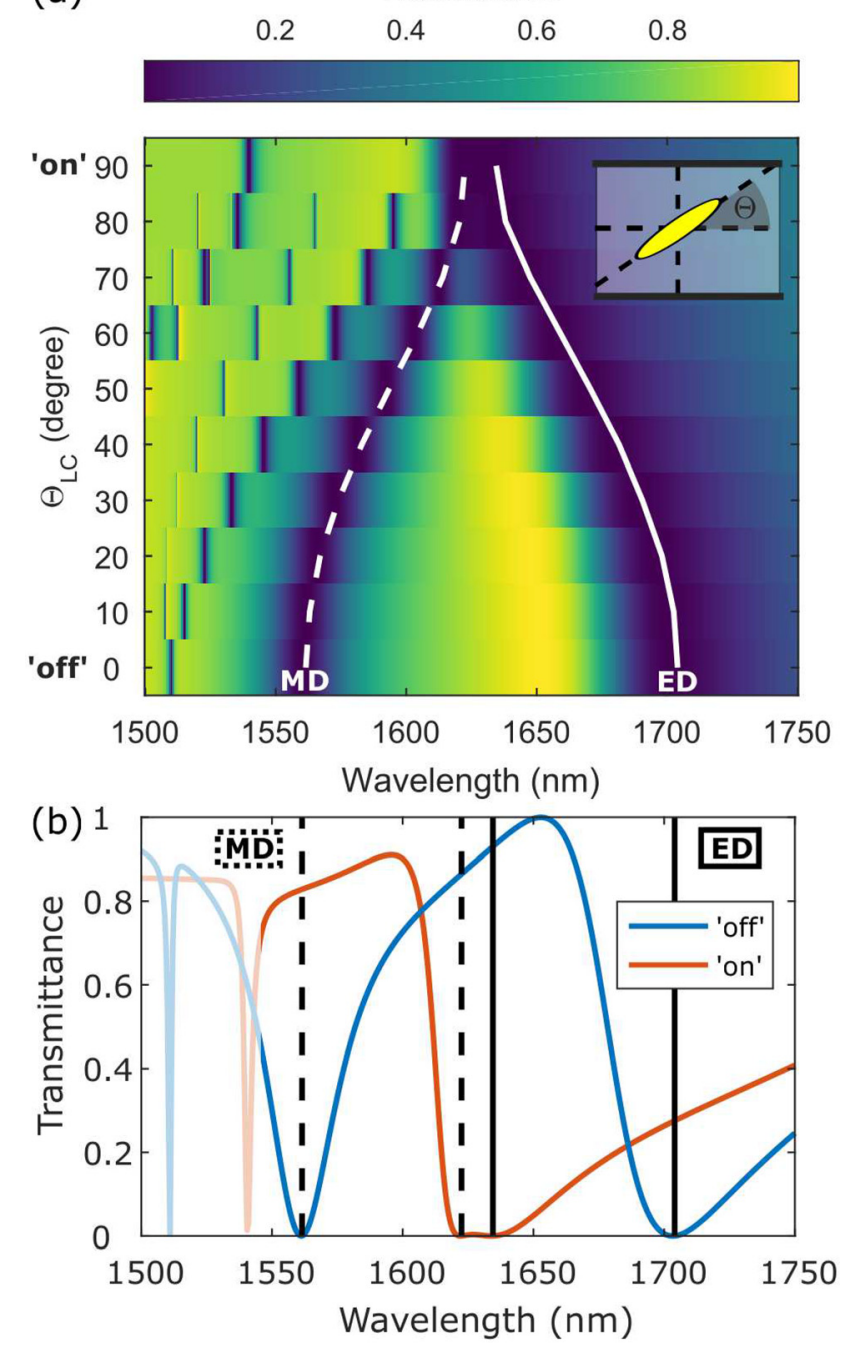

FIG. 2. (a) Simulation of the spectral resonance shifts expected for a complete reorientation of the LC surrounding the metasurface. $\Theta_{L C}$ denotes the angle between the metasurface plane and the LC anisotropy axis, with voltage "off" corresponding to $\Theta_{L C}=0^{\circ}$ and voltage "on" corresponding to $\Theta_{L C}=90^{\circ}$. White lines denote the spectral positions of the electric (solid, $\mathrm{ED}$ ) and magnetic (dashed, MD) resonance positions. The additional spectral features at small wavelengths correspond to lattice-type resonances. (b) Calculated transmittance spectra for $\Theta_{L C}=0^{\circ}$ (blue) and $\Theta_{L C}=90^{\circ}$ (red). The resonance positions of the electric (ED) and magnetic dipole (MD) resonances are indicated by the solid and dashed vertical lines, respectively.

for the "on" case. This can be explained by the imperfect alignment of the LC molecules in the vicinity of the metasurface. The measured absolute change in transmittance $\Delta T$ $=T(70 \mathrm{~V})-T(0 \mathrm{~V})$ for a transition from the "off" state to the "on" state is shown in Fig. 3(b) and reaches a maximum of $75 \%$. One can clearly see that the transmittance is switched from opaque to transparent at around $1540 \mathrm{~nm}$ and vice versa in the spectral region from $1590 \mathrm{~nm}$ to $1620 \mathrm{~nm}$. Note that in the literature instead of absolute transmittance (or reflectance) changes, some authors prefer to specify the achieved tuning or switching contrast $\Delta T / T_{0}$, which reaches up to $700 \%$ in our case for $T_{0}=T(0 \mathrm{~V})$. This high value can be attributed not only to the strong transmittance change but also to low transmission at resonance.

Finally, we directly measured the phase change introduced by the metasurface for different applied voltages at the
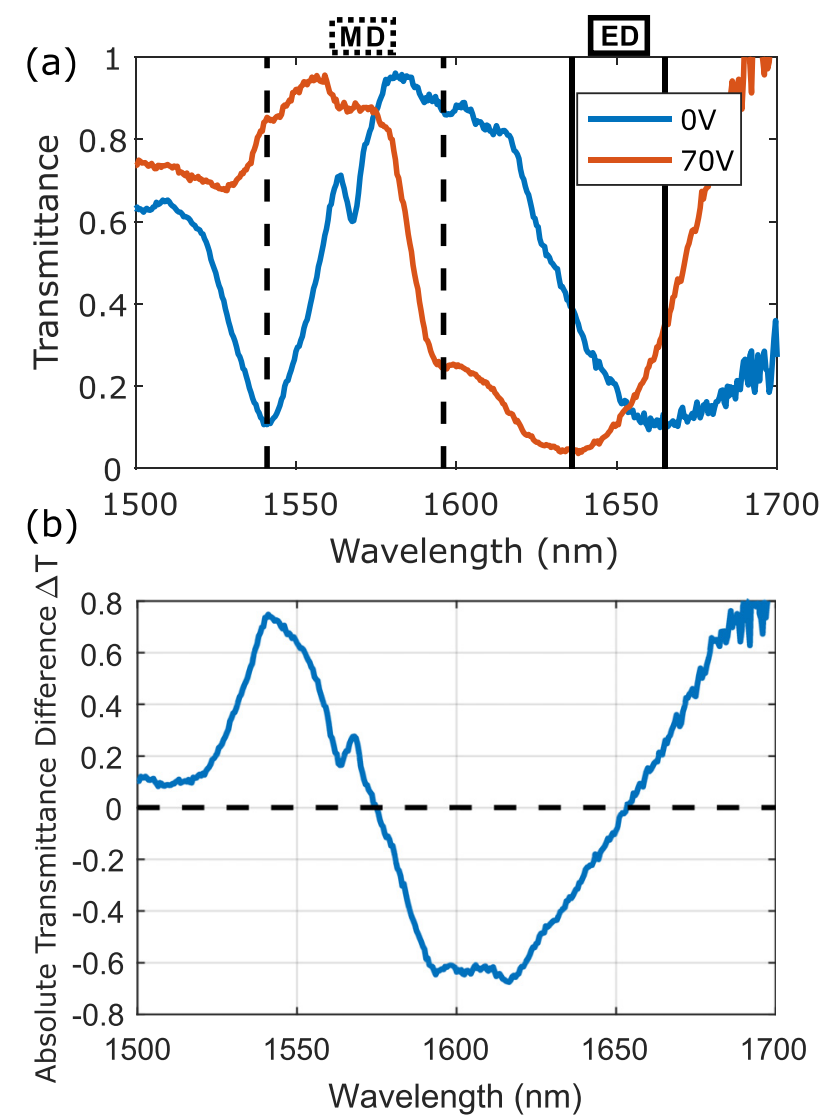

FIG. 3. (a) Measured transmittance spectra for $0 \mathrm{~V}$ (blue) and $70 \mathrm{~V}$ (red) bias voltages. The spectral positions of the electric (ED) and magnetic dipole (MD) resonances are indicated by the solid and dashed vertical lines, respectively. (b) Measured change in transmittance $\Delta T=T(70 \mathrm{~V})-T(0 \mathrm{~V})$ upon application of the bias voltage.

telecommunication wavelength of $1550 \mathrm{~nm}$. Using a homebuilt imaging Mach-Zehnder interferometer and the phase retrieval method previously described by Chong et al., ${ }^{4}$ we imaged the phase imprinted onto the incident light field upon transmission through the metasurface for a variation of the applied voltage from $0 \mathrm{~V}$ to $70 \mathrm{~V}$ in steps of $5 \mathrm{~V}$. The wavelength of the employed laser source is $\lambda=1550 \mathrm{~nm}$, which is close to the spectral position of the magnetic dipole resonance in the "off" case and in an off-resonant spectral regime of the metasurface for the "on" case. For a quantitative evaluation of the induced phase change, we average the phase values over the spatial extent of the metasurface as a function of applied voltage. These results are shown in Fig. 4(b). As expected for complete detuning of a single resonance away from the laser wavelength, we observe a phase change with a magnitude of up to $\pi$. The switching dynamics was mainly observed between $10 \mathrm{~V}$ and $30 \mathrm{~V}$ corresponding to the reorientation of the liquid crystals. While for a single resonance, the observed phase change is connected to a change in transmission intensity, the concept of silicon Huygens' metasurfaces employing overlapping electric and magnetic dipole resonances may open a way to achieve a phase change without changing the transmission intensity. ${ }^{3}$

In conclusion, we have demonstrated voltage tuning of the spectral positions of the electric and magnetic dipolar Mie-type resonances of an all-dielectric metasurface using the reorientation of the nematic liquid crystals by an external 
(a)
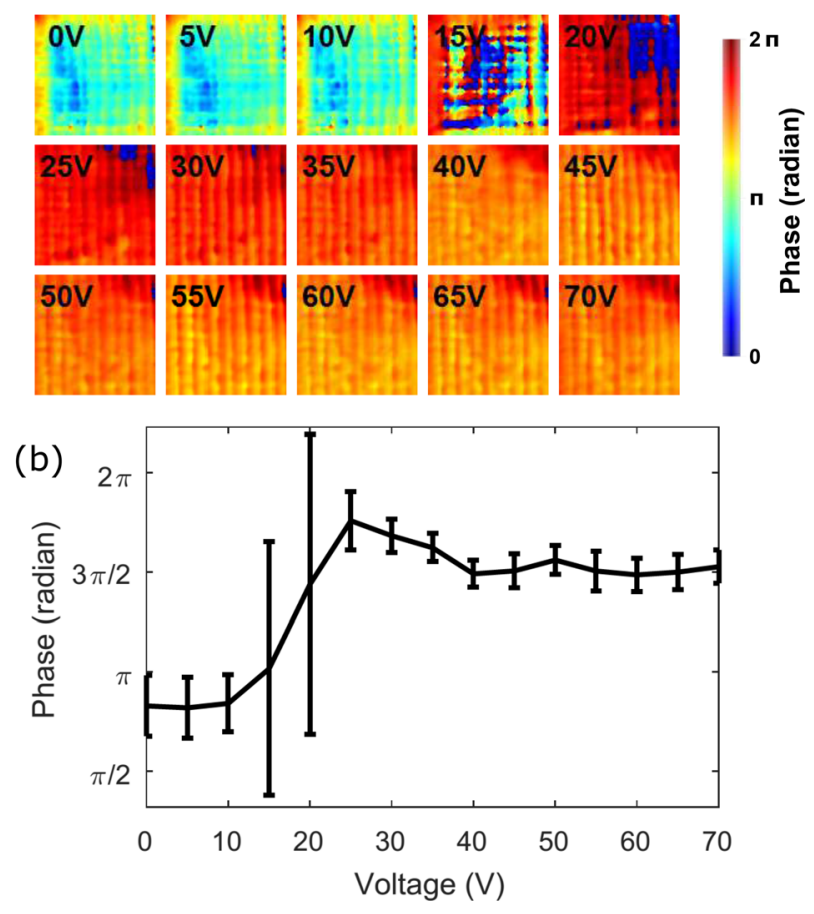

FIG. 4. (a) Measured transmission phase across the array of the LC metasurface for different biasing voltages at $\lambda=1550 \mathrm{~nm}$. (b) Phase accumulated by the light transmitted through the metasurface. The error bars indicate the spatial phase variation observed over the extent of the metasurface.

electric field. We achieved experimental transmission modulations of up to $75 \%$ and a phase change of up to approximately $\pi$. Our results pave the way for realizing dynamically reconfigurable metasurfaces with broad application prospects, including dynamic holography, tunable imaging, and active beam steering.

The authors acknowledge the financial support from the Australian Research Council and the Thuringian State Government within its ProExcellence initiative $\left(\mathrm{ACP}^{2020}\right)$. This work was performed, in part, at the Center for Integrated Nanotechnologies, an Office of Science User Facility operated for the U.S. Department of Energy (DOE) Office of Science. Sandia National Laboratories is a multiprogram laboratory managed and operated by Sandia Corporation, a wholly owned subsidiary of Lockheed Martin Corporation, for the U.S. Department of Energy's National Nuclear Security Administration under Contract No. DE-AC0494AL85000. The authors acknowledge their participation in the Erasmus Mundus NANOPHI project under Contract No. 2013 5659/002-001.
${ }^{1}$ L. Zou, W. Withayachumnankul, C. M. Shah, A. Mitchell, M. Bhaskaran, S. Sriram, and C. Fumeaux, Opt. Express 21, 1344 (2013).

${ }^{2}$ D. Lin, P. Fan, E. Hasman, and M. L. Brongersma, Science 345, 298 (2014).

${ }^{3}$ M. Decker, I. Staude, M. Falkner, J. Domínguez, D. N. Neshev, I. Brener, T. Pertsch, and Y. S. Kivshar, Adv. Opt. Mater. 3, 813 (2015).

${ }^{4}$ K. E. Chong, I. Staude, A. James, J. Domínguez, S. Liu, S. Campione, G. Subramania, T. S. Luk, M. Decker, D. N. Neshev, I. Brener, and Y. Kivshar, Nano Lett. 15, 5369 (2015).

${ }^{5}$ M. I. Shalaev, J. Sun, A. Tsukernik, A. Pandey, K. Nikolskiy, and N. M. Litchinitser, Nano Lett. 15, 6261 (2015).

${ }^{6}$ A. Arbabi, Y. Horie, M. Bagheri, and A. Faraon, Nat. Nanotechnol. 10, 937 (2015).

${ }^{7}$ Y. F. Yu, A. Y. Zhu, R. Panlagua-Dominguez, Y. H. Fu, B. Luk'yanchuk, and A. I. Kuznetsov, Laser Photonics Rev. 9, 412 (2015).

${ }^{8}$ K. E. Chong, L. Wang, I. Staude, A. James, J. Dominguez, S. Liu, G. S. Subramania, M. Decker, D. N. Neshev, I. Brener, and Y. S. Kivshar, ACS Photonics 3, 514 (2016).

${ }^{9}$ I. Staude, A. E. Miroshnichenko, M. Decker, N. T. Fofang, S. Liu, E. Gonzales, J. Dominguez, T. S. Luk, D. N. Neshev, I. Brener, and Y. S. Kivshar, ACS Nano 7, 7824 (2013).

${ }^{10}$ S. Liu, M. B. Sinclair, T. S. Mahony, Y. C. Jun, S. Campione, J. Ginn, D. A. Bender, J. R. Wendt, J. F. Ihlefeld, P. G. Clem, J. B. Wright, and I. Brener, Optica 1, 250 (2014).

${ }^{11}$ C. Wu, N. Arju, G. Kelpp, J. Fan, J. Dominguez, E. Gonzales, E. Tutuc, I. Brener, and G. Shvets, Nat. Commun. 5, 3892 (2014).

${ }^{12}$ P. Moitra, B. A. Slovick, Z. G. Yu, S. Krishnamurthy, and J. Valentine, Appl. Phys. Lett. 104, 171102 (2014).

${ }^{13}$ Y. Yang, I. I. Kravchenko, D. P. Briggs, and J. Valentine, Nat. Commun. 5, 5753 (2014).

${ }^{14}$ M. Khorasaninejad, W. Zhu, and K. B. Crozier, Optica 2, 376 (2015).

${ }^{15}$ N. I. Zheludev and Y. S. Kivshar, Nat. Mater. 11, 917 (2012).

${ }^{16}$ A. I. Kuznetsov, A. E. Miroshnichenko, M. L. Brongersma, Y. S. Kivshar, and B. Luk'yanchuk, Science 354, 2472 (2016).

${ }^{17}$ G. Mie, Ann. Phys. 330, 377 (1908).

${ }^{18}$ A. B. Evlyukhin, S. M. Novikov, U. Zywietz, R. L. Eriksen, C. Reinhardt, S. I. Bozhevolnyi, and B. N. Chichkov, Nano Lett. 12, 3749 (2012).

${ }^{19}$ A. I. Kuznetsov, A. E. Miroshnichenko, Y. H. Fu, J. Zhang, and B. Luk'yanchuk, Sci. Rep. 2, 492 (2012).

${ }^{20}$ J. Sautter, I. Staude, M. Decker, E. Rusak, D. N. Neshev, I. Brener, and Y. S. Kivshar, ACS Nano 9, 4308 (2015).

${ }^{21}$ P. Gutruf, C. Zou, W. Withayachumnankul, M. Bhaskaran, S. Sriram, and C. Fumeaux, ACS Nano 10, 133 (2016).

${ }^{22}$ S. M. Kamali, E. Arbabi, A. Arbabi, Y. Horie, and A. Faraon, Laser Photonics Rev. 10, 1002-1008 (2016).

${ }^{23}$ A. Karvounis, B. Gholipour, K. F. MacDonald, and N. I. Zheludev, Appl. Phys. Lett. 109, 051103 (2016).

${ }^{24}$ J. A. Bossard, X. Liang, L. Li, S. Yun, D. H. Werner, B. Weiner, T. S. Mayer, P. F. Cristman, A. Diaz, and I. C. Khoo, IEEE Trans. Antennas Propag. 56, 1308 (2008).

${ }^{25}$ M. R. Shcherbakov, P. P. Vabishchevich, A. S. Shorokhov, K. E. Chong, D.-Y. Choi, I. Staude, A. E. Miroshnichenko, D. N. Neshev, A. A. Fedyanin, and Y. S. Kivshar, Nano Lett. 15, 6985 (2015).

${ }^{26}$ S. Makarov, S. Kudryashov, I. Mukhin, A. Mozharov, V. Milichko, A. Krasnok, and P. Belov, Nano Lett. 15, 6187 (2015).

${ }^{27}$ D. G. Baranov, S. V. Makarov, V. A. Milichko, S. I. Kudryashov, A. E. Krasnok, and P. A. Belov, ACS Photonics 3, 1546-1551 (2016).

${ }^{28}$ Y.-W. Huang, H. W. H. Lee, R. Sokhoyan, R. Pala, K. Thyagarajan, S. Han, D. P. Tsai, and H. A. Atwater, Nano Lett. 16, 5319-5325 (2016).

${ }^{29}$ J. Li, S.-T. Wu, S. Brugioni, R. R. Meucci, and S. Faetti, J. Appl. Phys. 97, 073501 (2005).

${ }^{30}$ W. Helfrich, J. Chem. Phys. 51, 4092-4105 (1969).

${ }^{31}$ R. Chen, Liquid Crystal Displays: Fundamental Physics and Technology, Wiley Series in Display Technology (Wiley, 2011). 